\title{
Fibromatosis gingival hereditaria una inusual enfermedad genética: reporte de caso
}

\author{
Hereditary gingival fibromatosis a rare genetic disease: \\ case report
}

\author{
Harris Ricardo J1, López Álvarez A², Martínez Rangel J³, Martínez Soto R³
}

\begin{abstract}
RESUMEN
La fibromatosis gingival hereditaria es una enfermedad poco frecuente, asociada a factores genéticos, que se caracterizada por aumento en el tamaño del tejido gingival, el cual genera dificultades emocionales, estéticas y funcionales. En el presente artículo se reporta un caso de una paciente femenina de 13 años con aumento generalizado en el volumen de la encía, que cubre casi todos los dientes, la historia familiar fue muy importante para el diagnóstico de fibromatosis gingival hereditaria, ya que la madre y un hermano presentaron la misma manifestación.
\end{abstract}

Rev. Clin. Periodoncia Rev. Clin. Periodoncia Implantol. Rehabil. Oral Vol. 5(2); 87-89, 2012.

Palabras clave: Fibromatosis gingival, herencia, gingivectomía, niño (Decs Bireme).

\section{ABSTRACT}

Hereditary gingival fibromatosis is a rare disorder, associated with genetic factors, characterized by various degrees of attached gingival overgrowth, which generates emotional, aesthetic and functional disorders. This article reports the case of a 13-year-old female who presented a generalized severe gingival overgrowth, involving the maxillary and mandibular arches and covering almost the whole dentition. The family history was very important for the diagnosis of hereditary gingival fibromatosis, as the mother and a brother had the same disorder.

Rev. Clin. Periodoncia Implantol. Rehabil. Oral Vol. 5(2); 87-89, 2012.

Key words: Fibromatosis gingival, heredity, gingivectomy, child (Mesh Database).

\section{INTRODUCCIÓN}

La fibromatosis gingival hereditaria $(\mathrm{FGH})$ es una enfermedad genéticamente heterogénea, proliferativa, de carácter benigno, con una incidencia de 1 en 750.000 , es de forma autosómica dominante y se caracteriza por aumento de forma generalizada del volumen de la encía, llegando a cubrir las coronas de los dientes ${ }^{(1)}$; se presenta de forma aislada, con antecedentes familiares de la misma condición, no se encuentra asociada a síndromes, fármacos o placa dental(2). Es importante señalar que ciertos fármacos como los anticonvulsivantes, bloqueadores de los canales de calcio, ciclosporina A, entre otros y algunos síndromes entre ellos Cowden, Rutherford, Murray-Puretic-Drescher y Cross, están relacionados con el crecimiento gingival(3).

En cuanto a la etiología Pampel et al., en un estudio de ligamiento localizaron lugares de herencia autosómica dominante no sindrómica en el cromosoma 2, específicamente en el 2p21-p22 y 2p22.3-p23.3; el cromosoma 2 es el segundo cromosoma humano, que abarca más de 243 millones de pares de bases en la construcción del ADN y que representa casi el $8 \%$ del ADN total de las células ${ }^{(4)}$.

Hart et al., en un estudio identificaron una mutación del gen SOS1 y afirman que un solo nucleótido por inserción en el codón 1083 del gen SOS1 es la causa de la mutación en la FGH en los seres humanos, el gen se encuentra situado en el brazo corto del cromosoma 2p21-2p22 y codifica una proteína que es un factor de intercambio de nucleótidos de guanina para las proteínas RAS, las proteínas de membrana que se unen a nucleótidos de guanina y participar en las vías de transducción de señales ${ }^{(5)}$.

\section{CARACTERISTTICAS CLÍNICAS}

Clínicamente la afección gingival es generalizada, el tejido es de aspecto fibroso, consistencia firme, no sangrante, asintomática, no tiene predilección por genero sexual, se manifiesta con la erupción de la dentición decidua o permanente ${ }^{(6)}$; el crecimiento gingival origina problemas funcionales y estéticos, como desplazamiento dental, diastemas, dificultades fonéticas, en el proceso de la masticación, retraso en la erupción dentaria, caries y enfermedad periodontal, los cuales están relacionados con la retención de placa bacteriana generada por el agrandamiento gingival y efectos en el desarrollo emocional y sicológico ${ }^{(7)}$. Puede presentarse asociada a hipertricosis, retraso mental y epilepsia, pero en ocasiones, la única manifestación clínica es el agrandamiento gingival(8).

Kather et al., en un estudio examinaron las características histomorfológicas e histomorfométricas en muestras de tejido de tres familias con $\mathrm{FGH}$, en los resultados reportan que la presencia de haces de colágeno en el tejido conectivo y fibroblastos ${ }^{(9)}$; Kelekis et al., también describen que se observa a nivel histológico paquetes densos grueso de colágeno y fibroblastos en el conectivo, destacando pequeñas partículas calcificadas y pequeños focos óseos ${ }^{(10)}$.

Baptista afirma que el procedimiento quirúrgico con gingivectomía es una buena alternativa terapéutica donde se disminuye el grosor y altura de los tejidos gingivales ${ }^{(11)}$. Deangelo et al., realizaron una revisión de la literatura referente a la FGH y describen que la gingivectomía es la técnica quirúrgica adecuada para el tratamiento y resaltan que restaurar el contorno

1. Odontólogo. Universidad del Sinú Cartagena. Especialista en Estomatología y Cirugía Oral, Universidad de Cartagena. Candidato a Magister en Microbiología Molecular, Universidad Libre. Docente Corporación Universitaria Rafael Núñez. Colombia.

2. Odontólogo. Universidad de Cartagena. Especialista en Implantología Oral, Universidad Católica Argentina. Docente Corporación Universitaria Rafael Núñez. Colombia.

3. Estudiantes de Odontología. Corporación Universitaria Rafael Núñez. Colombia.

Correspondencia autor: Jonathan Harris Ricardo. j.harris.r@hotmail.com. Programa de Odontología Corporación Universitaria Rafael Núñez. Centro Avenida Escallón Pasaje la Moneda Local 111. Cartagena. D.T. y C. Colombia. Trabajo recibido el 05/03/2012. Aceptado para su publicación el 03/05/2012. 
gingival fisiológico permite un mejor control de placa bacteriana, lo cual podría mejorar los resultados a largo plazo o disminuir la recurrencia ${ }^{(12)}$. Taj y cols., reportaron un caso de un niño con FGH en el cual describen que puede ser necesario repetir el tratamiento quirúrgico debido a la alta recurrencia y que esta generalmente ocurre dentro de los 2 primeros años posteriores a la gingivectomía ${ }^{(13)}$.

Aunque la FGH es una patología poco frecuente, es importante que el odontólogo conozca la fisiopatología de la enfermedad y el compromiso hereditario, para establecer un diagnóstico precoz y una derivación oportuna, con el fin de evitar futuras complicaciones.

\section{REPORTE DEL CASO}

Paciente femenina remitida al Servicio de Estomatología y Cirugía Oral de la Clínica Odontológica de la Corporación Universitaria Rafael Núñez por presentar incremento generalizado en el tamaño del tejido gingival.

En la anamnesis relata tener 13 años de edad, el crecimiento gingival progresivo y lo padece desde hace un año aproximadamente, afectando el maxilar y la mandíbula, la madre describe que esta es la segunda vez que presenta aumento en el tamaño de la encía, ya que a los 4 años de edad, presentó la primera manifestación y se le realizó gingivectomía obteniendo buenos resultados; también resalta que ella y un hermano de la paciente presentaron la misma condición; el desarrollo sicomotor es normal, en la revisión de órganos y sistemas no presentó alteraciones.

En la exploración Intrabucal se observó agrandamiento gingival generalizado que cubría más de dos tercios de la corona dental, de consistencia firme, textura fibromatosa, no sangrante, asintomático, originando discapacidad funcional y estética (Figuras 1 y 2 ). En el examen radiográfico se observó en la ortopantomografía dientes en buen estado general y la presencia del diente número 73 (Figura 3). Se estableció diagnóstico de fibromatosis gingival hereditaria teniendo en cuenta los parámetros clínicos y antecedentes familiares.

Se realizó interconsulta con medicina pediátrica el cual efectúa valoración, ordenó exámenes de laboratorio que incluyeron hemograma completo, extendido de sangre periférica, creatinina, nitrógeno ureico, transaminasas como GOT y GPT los cuales se encontraron entre los valores normales, descartando trastornos hemáticos, renales y hepáticos, reporta que no hallar relación con síndromes, encontrándola en buena condición física, psicomotora y sistémica.

\section{TRATAMIENTO}

Previo a la firma del consentimiento informado se le explicaron las posibles complicaciones, se procedió a la realización de gingivectomía y gingivoplastía como técnicas quirúrgicas, la terapéutica fue bajo anestesia local administrando lidocaína al 2\% con epinefrina 1:80.000, inicialmente se realizó el procedimiento quirúrgico en la mandíbula y 21 días después en el maxilar.

La técnica consistió inicialmente en una incisión a bisel interno a un ángulo de $45^{\circ}$ grados hacia el ápice de los dientes, posteriormente se efectuó incisión intrasurcular creando un collar de tejido gingival el cual fue removido subsiguientemente (Figura 4) y se continuó con reafinado de las áreas interproximales; luego se precedió con la hemostasia y colocación de un tutor en acetato con cemento quirúrgico periodontal, se ordenó farmacoterapia que incluyó Amoxal capsulas de 500 mg, como antibiótico y Motrin tabletas de $600 \mathrm{mg}$, por su efecto analgésico y antiinflamatorio, recomendaciones sobre una adecuada higiene oral y clorhexidina al $0.12 \%$ como enjuague bucal.

El examen histopatológico describe microscópicamente fragmentos de mucosa oral revestidos por epitelio paraqueratósico, hiperplásico, sobre estroma con abundante haces gruesos de colágeno dispuestos al azar, compatible con fibromatosis gingival.

El postoperatorio fue satisfactorio y se programaron tres controles postquirúrgicos con intervalos de 7 días por cada procedimiento (maxilar y mandíbula), en el primer control se retiró el tutor quirúrgico y se observó disminución en el volumen del tejido gingival, exposición de las coronas dentarias, zonas de color rojo a nivel gingival como consecuencia del procedimiento quirúrgico, indicando el inicio de las primeras fases de la cicatrización y manifestó molestias al momento de la alimentación, en el segundo y tercer control se evidenció buena evolución del proceso de cicatrización, contorno y morfología adecuada de las encías, el color gingival paso de un tono rojo a rosa pálido, mayor exposición de las coronas dentales (Figura 5), expresó mejoras en el proceso de masticación y mayor relación interpersonal en la escuela.
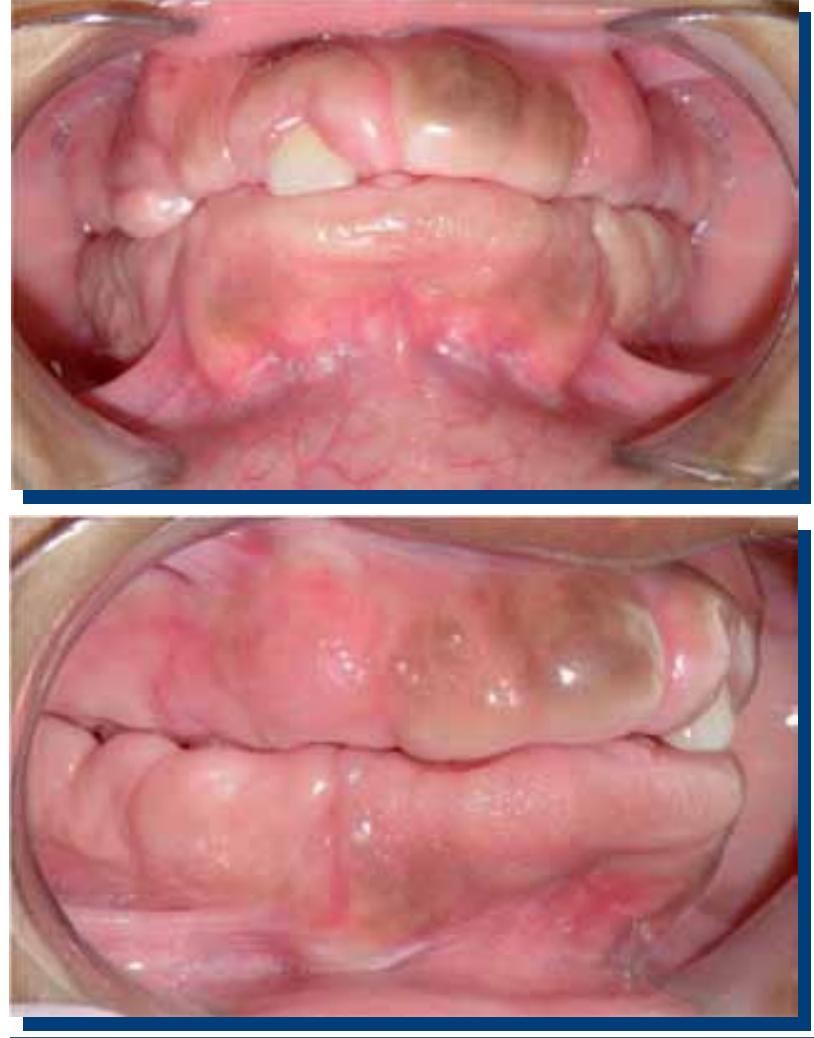

Figuras 1 y 2. Aumento en el volumen de la encía de forma generalizada, con apariencia fibromatosa, cubriendo la corona de los dientes.

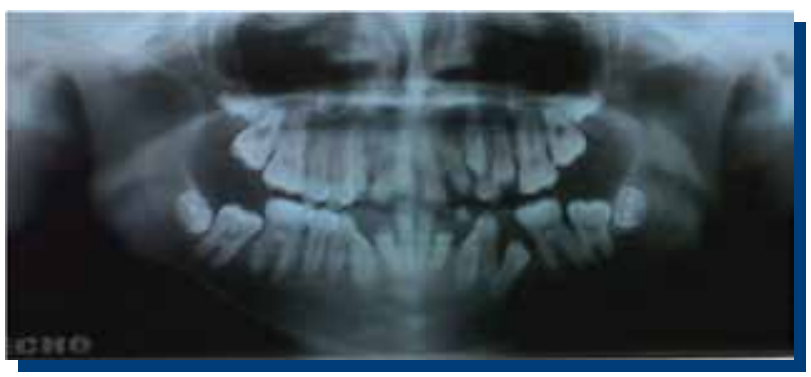

Figura 3. Ortopantomografía donde se observa presencia del diente 73 retenido.

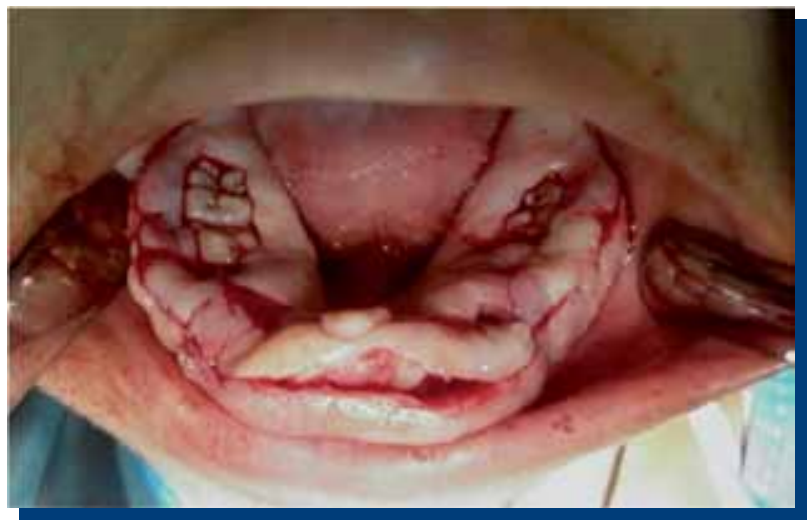

Figura 4. Incisión a bisel interno.

\section{DISCUSIÓN}

La fibromatosis gingival es una patología benigna, que se manifiesta con aumento en el volumen de la encía, de forma generalizada, dificultando la higiene bucal, el habla, es de crecimiento lento y asintomática ${ }^{(14)}$. Saygun et al. y Häkkinen et al., afirman que la FGH se muestra clínicamente como un aumento en el volumen de la 


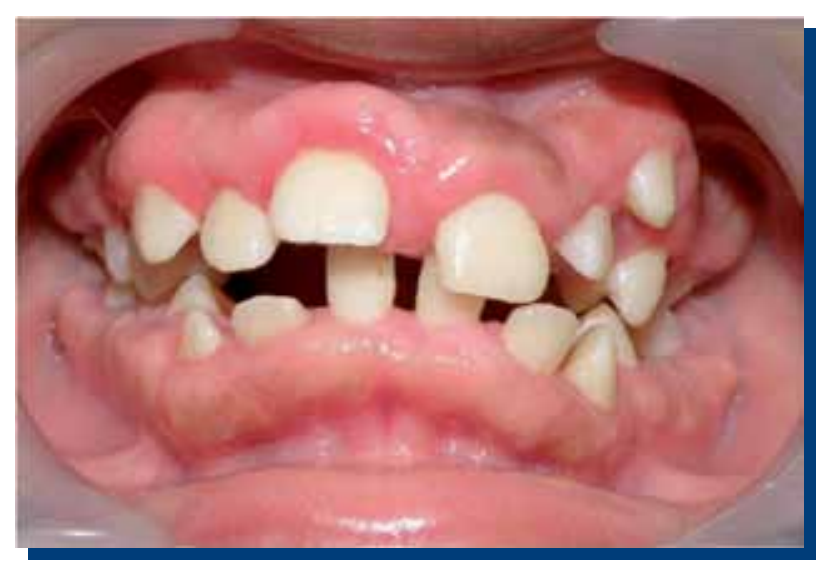

Figura 5. Control clínico donde se observó disminución en el volumen del tejido gingival, con exposición de las coronas dentarias.

encía, de forma generalizada, que puede llegar a cubrir las coronas de los dientes, generando alteraciones bucales como diastemas, dificultad en la masticación y retraso en la erupción dentaria ${ }^{(15,16)}$; características clínicas similares a las reportadas en el presente caso donde se observó agrandamiento gingival generalizado en el maxilar y mandíbula, cubriendo la totalidad de la corona de los dientes anteriores y más del $85 \%$ en los dientes posteriores, originando discapacidad funcional como incompetencia labial, problemas en el proceso de la masticación, retraso en la erupción de los dientes permanentes y dentición temporal tardía.

Bittencourt et al., en una revisión de la literatura sobre FGH reportaron que el compromiso estético importante y ocasiona dificultades psicosociales ${ }^{(17)}$, concordando con el presente caso de una niña con FGH que mostró un excesivo agrandamiento gingival generalizado, que cubría más de dos tercios de la corona de los dientes afectando la estética, el desarrollo de la autoestima, generando problemas sicológicos y emocionales, la paciente tenía dificultades en las relaciones interpersonales con los compañeros de la escuela, siempre se le notó triste y muy poco sonreía.

Ramakrishnan et al., reportaron un caso de paciente con FGH severa, en el examen radiográfico observaron la presencia de dientes temporales $54,64,75$ y 85 retenidos, afirman que los dientes deciduos retenidos por el tejido gingival fibromatoso es común(18); coincidiendo con el presente reporte el cual se observó el del diente 73 en la ortopantomografía, dicho diente se encuentra retenido por el tejido fibromatoso.

Odessey et al., en un estudio realizaron gingivectomía en tres pacientes con FGH severa en dos etapas primero resección en el maxilar y luego en la mandíbula, cambiando el método tradicional por cuadrantes, o sea cuatro etapas, obteniendo buenos resultados y sin recurrencia en un seguimiento por 18 meses $^{(19)}$. Ramakrishnan et al., reportaron el manejo multidisciplinario de un paciente con $\mathrm{FGH}$, con un seguimiento por un año, como terapéutica realizaron gingivectomía en todos los cuadrantes, obteniendo un peso de $160 \mathrm{gr}$ del tejido extirpado, ordenaron Amoxicilina e Ibuprofeno como terapia farmacológica y enjuagues con Clorhexidina, resaltando la gingivectomía como técnica quirúrgica adecuada, ya que se mostraron buenos resultados en el proceso de cicatrización, mejorando la función y estética(18); ${ }^{(18 i n c i d i e n d o ~}$ con el caso reportado en el que se realizó gingivectomía y gingivoplastía como tratamiento quirúrgico, se prescribió tratamiento farmacológico con Amoxal (Amoxicilina) y Motrin (Ibuprofeno), recomendando el uso de enjuagues con Clorhexidina al $0.12 \%$, los resultados postquirúrgicos fueron los esperados observando buena cicatrización de los tejidos periodontales, mejoras en el proceso de masticación, buena estética y no presenta recidiva en 8 meses de seguimiento.

\section{CONFLICTOS DE INTERÉS}

Los autores declaran no tener conflictos de interés.

\section{REFERENCIAS BIBLIOGRÁFICAS}

1. Avelar RL, de Luna Campos GJ, de Carvalho Bezerra Falcão PG, da Costa Araújo FA, de Carvalho RW, de Souza Andrade ES. Hereditary gingival fibromatosis: A report of four cases in the same family. Quintessence Int, 2010 Feb; 41(2): 99-102.

2. Vishnoi SL. Hereditary gingival fibromatosis: Report of four generation pedigree. International Journal of Case Reports and Images, 2011; 2(6): 1-5.

3. Coletta RD, Graner E. Hereditary gingival fibromatosis: A systematic review. J Periodontol, 2006 May; 77(5): 753-764.

4. Pampel M, Maier S, Kreczy A, Weirich-Schwaiger H, Utermann G, Janecke AR. Refinement of the GINGF3 locus for hereditary gingival fibromatosis. Eur J Pediatr, 2010 Mar; 169(3): 327-332.

5. Hart TC, Zhang Y, Gorry MC, Hart PS, Cooper M, Marazita ML, Marks JM, Cortelli JR, Pallos D. A mutation in the SOS1 gene causes hereditary gingival fibromatosis type 1. Am J Hum Genet, 2002 Apr; 70(4): 943-954.

6. Breen GH, Addante R, Black CC. Early onset of hereditary gingival fibromatosis in a 28-month-old. Pediatr Dent, 2009 Jul-Aug; 31(4): 286-288.

7. Sengün D, Hatipoğlu H, Hatipoğlu MG. Long-term uncontrolled hereditary gingival fibromatosis: A case report. J Contemp Dent Pract, 2007 Jan 1; 8(1): 90-96.

8. Wagaiyu EG, Ng'ang'a RN, Kemoli AM. Hereditary gingival fibromatosis: Report of family case series. East Afr Med J, 2009 Oct; 86(10): 491-493

9. Kather J, Salgado MA, Salgado UF, Cortelli JR, Pallos D. Clinical and histomorphometric characteristics of three different families with hereditary gingival fibromatosis. Oral Surg Oral Med Oral Pathol Oral Radiol Endod, 2008 Mar; 105(3): 348-352.

10. Kelekis-Cholakis A, Wiltshire WA, Birek C. Treatment and longterm follow-up of a patient with hereditary gingival fibromatosis: A case report. J Can Dent Assoc, 2002 May; 68(5): 290-294.
11. Baptista IP. Hereditary gingival fibromatosis: A case report. J Clin Periodontol, 2002 Sep; 29(9): 871-874.

12. DeAngelo S, Murphy J, Claman L, Kalmar J, Leblebicioglu B. Hereditary gingival fibromatosis a review. Compend Contin Educ Dent, 2007 Mar; 28(3): 138-143.

13. Taj-Taj $P$, Dolmestch $C$. Fibromatosis gingival hereditaria en niños. Caso clínico. Rev Clin Periodoncia Implantol Rehabil Oral, 2008; 1(2): 66-69.

14. Martelli-Junior H, Lemos DP, Silva CO, Graner E, Coletta RD. Hereditary gingival fibromatosis: Report of a five-generation family using cellular proliferation analysis. J Periodontol, 2005 Dec; 76(12): 2299-2305

15. Saygun I, Ozdemir A, Günhan O, Aydintuğ YS, Karslioğlu Y Hereditary gingival fibromatosis and expression of Ki-67 antigen: A case report. J Periodontol, 2003 Jun; 74(6): 873-878.

16. Häkkinen L, Csiszar A. Hereditary gingival fibromatosis: Characteristics and novel putative pathogenic mechanisms. J Dent Res, 2007 Jan; 86(1): 25-34.

17. Bittencourt LP, Campos V, Moliterno LF, Ribeiro DP, Sampaio RK. Hereditary gingival fibromatosis: Review of the literature and a case report. Quintessence Int, 2000 Jun; 31(6): 415-418.

18. Ramakrishnan $T$, Kaur M. Multispeciality approach in the management of patient with hereditary gingival fibromatosis: 1-year followup: A case report. Int J Dent, 2010: 575979. Epub 2010 Dec 23. 19. Odessey EA, Cohn AB, Casper F, Schechter LS. Hereditary gingival fibromatosis: Aggressive 2-stage surgical resection in lieu of traditional therapy. Ann Plast Surg, 2006 Nov; 57(5): 557-560. 